\title{
Modelling and Control of a Tilt-Wing Unmanned Aerial Vehicle
}

\author{
E. Small, E. Fresk, G. Andrikopoulos, and G. Nikolakopoulos
}

\begin{abstract}
In this article a Tilt-Wing Unmanned Aerial Vehicle (TW-UAV) and the preliminary evaluation of its hovering characteristics in extended simulation studies are presented. In the beginning, an overview of the TW-UAV's design properties are established, highlighting the novelties of the proposed structure and the overall merits. The TW-UAV's design and structural properties are mathematically modeled and utilized for the synthesis of a cascaded P-PI and PID based control structure for the regulation of its hovering performance. In addition, extensive simulation trials are performed in order to evaluate the structure's efficiency in controlling the TW-UAV's attitude and position under various noise and disturbance scenarios.
\end{abstract}

\section{INTRODUCTION}

The area of Unmanned Aerial Vehicles (UAVs) and more specifically the related research specialized in multirotors has recently seen rapid growth, mainly due to their efficiency in accomplishing complex missions and flight scenarios [1]. The vast application range of this type of UAVs includes search and rescue missions, forest fire surveillance, border interdiction, area exploration and mapping, agricultural services, buildings inspection, marine operations, media coverage, and many more e.g. in [2].

Generally, multirotors do not possess the energy efficiency of a fixed wing aircraft for long-distance flight, since the research focus is mainly placed on their Vertical Take-Off and Landing (VTOL) properties rather than the utilization of wings to produce a lifting force. Thus, the goal of developing an aircraft with long-distance efficiency and VTOL capabilities recently gave rise to the tilt-rotor or tilt-wing aircraft, which is a combination of a multirotor and a fixedwing aircraft [3], [4], [5], [6].

The primary novelty presented in this article stems from the formulation of an innovative modelling and control scheme for an optimally designed novel Tilt-Wing UAV (TW-UAV). With regards to the optimality of the design, the interested reader can directly refer to [3], while in this article, for consistency, the main characteristics of the second revision of the design mentioned in [3] will be presented in detail. A tilt-wing instead of a tilt-rotor design was chosen for this type of aircraft, since rotating the wing in parallel with the motor while transitioning between flying and hovering modes, provides important mechanical advantages. Specifically, the drag caused by a propeller partially causing

The authors are with the Control Engineering Group, Department of Computer Science, Electrical and Space Engineering, Luleå University of Technology, SE-97187, Luleå, Sweden

This work has received funding from the European Union's Horizon 2020 Research and Innovation Programme under the Grant Agreement No.644128, AEROWORKS.

Corresponding Author's email: geonikeltu.se (C) 2016 IEEE air flow over the wing is minimized, and this seemingly small difference in energy consumption proves crucial when it comes to the development of small-scale UAVs, where the available power is hugely limited due to their design characteristics [7]. In addition, having fewer moving parts reduces the complexity of the physical structure as well as that of the mathematical model. This in turn translates into simpler control structures. Thus it is advantageous to base the design on a previously built prototype [3] so that more precise improvements can be evaluated, whilst existing experimental data can still be applied.

The second novelty presented in the article comes from the proposed modelling and control scheme based on conventional algorithms for the preliminary evaluation of the hovering characteristics of this innovative design. For this purpose, a cascade P-PI controller is utilized to control the attitude of the aircraft, whilst multiple PID loops are used for the position control.

The rest of the article is structured as follows: Section II presents the design specifications of the proposed aircraft; in Section III the dynamic equations of motion are derived; the control structure utilized for the attitude and position control is presented in Section IV; in Section V the preliminary results of the proposed UAV's hovering properties are evaluated through extensive simulation trials; and finally, the conclusions are drawn in Section VI.

\section{DESIGN PROPERTIES}

The proposed TW-UAV was initially based on the design presented in [3]. The graphical representation of the novel TW-UAV with all the components of interest highlighted is depicted in Figure 1.

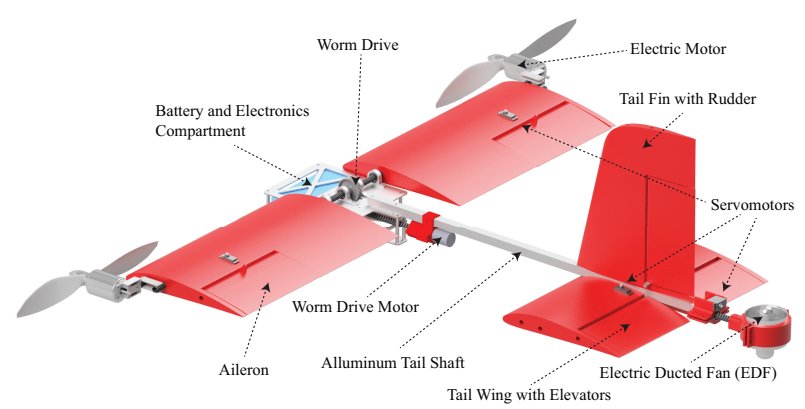

Fig. 1. Graphical representation of the TW-UAV with components of interest highlighted.

For consistency with [3], in the formulation of the modelling and control framework, each major structural part is 


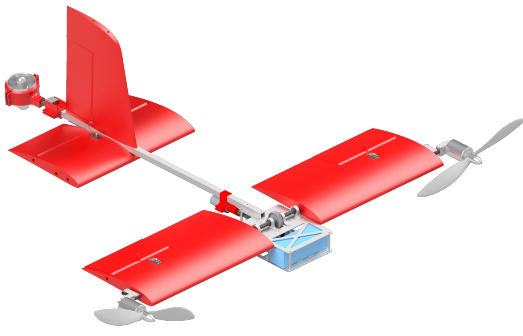

(a)

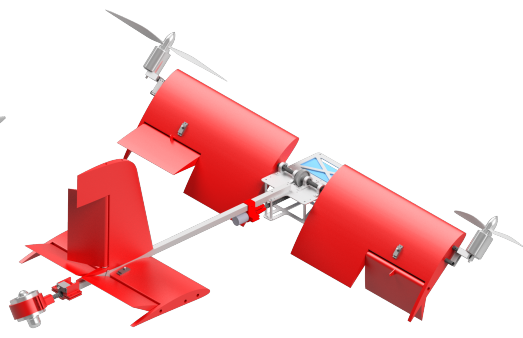

(b)

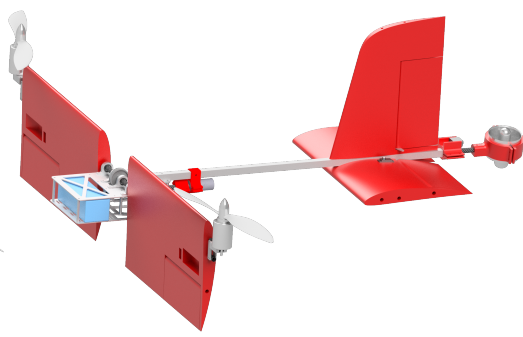

(c)

Fig. 2. Graphical representation of the TW-UAV during (a) flying, (b) dynamic, and (c) hovering states.

described below and any modifications in the revised version of the adopted design for the TW-UAV are discussed.

Given the complexities introduced when switching between the two flight modes (VTOL and plane modes), a design with as few moving parts as possible, while maintaining structural rigidity and stability, is desirable. One solution to this problem is to have the main wings and propellers joined as one structure that can rotate around an axis perpendicular to the chassis of the aircraft as indicated in Figure 2.

In addition, the wing structure is both rotated and held in place by a swiveling mechanism called a worm drive as indicated in Figure 3. In this case, an electric motor drives the worm, which in turn rotates a worm gear to which the entire wing structure is attached. The motor can rotate the wings, but the mechanics of the drive prevent any other rotational forces to be transferred the wings.

The main chassis of the TW-UAV, i.e. the aluminum structure holding the worm drive, the battery, and the electronic components, is designed to hold the worm drive in place effectively, while providing ample space for the battery and electronics.

A fundamental complication in the prototype design of the TW-UAV [3] was that the tail experienced excessive vibration and wobbling. This effect could be traced to the fact that the tail shaft was cylindrical and therefore prone to twisting and bending in an undesirable manner. This problem has been solved by exchanging the cylindrical carbon fiber pole with a hollow aluminum shaft with a square profile. This easily implemented exchange provides a sturdier and more

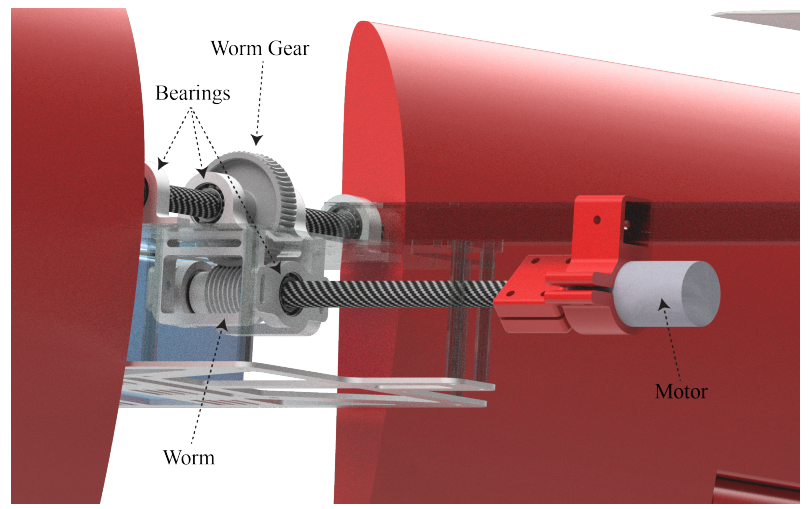

Fig. 3. Graphical detail of the worm-drive mechanism. efficiently assembled tail section.

The most noticeable change made to the prototype design is the size and width of the wing surfaces. A longer inlength airfoil is utilized, which allows the shortening of the wingspan in order to add mechanical stability, while increasing lift capabilities of all the wing surfaces.

Most conventional airplane structures are constructed with a tail wing that generally produces down force to stabilize the tail since the Centre of Mass (COM) is located near, or in front of, the centre of lift [8]. In the proposed TW-UAV design, the COM is behind the main wings so as to ensure hovering capabilities, resulting in the tail structure having a substantial relative weight with respect to the rest of the aircraft. For this reason, the tail wings are constructed to provide lift for the entire tail section by utilizing the same wing profile as the main wings while simultaneously having elevators that can vary this lift significantly during flight so as to provide elevation control capabilities.

\section{SySTEM MODELING}

This section presents the mathematical model for the hovering mode of the TW-UAV. Firstly, the force and torque equations are derived from the acting force diagram presented in Figure 4 as shown below.

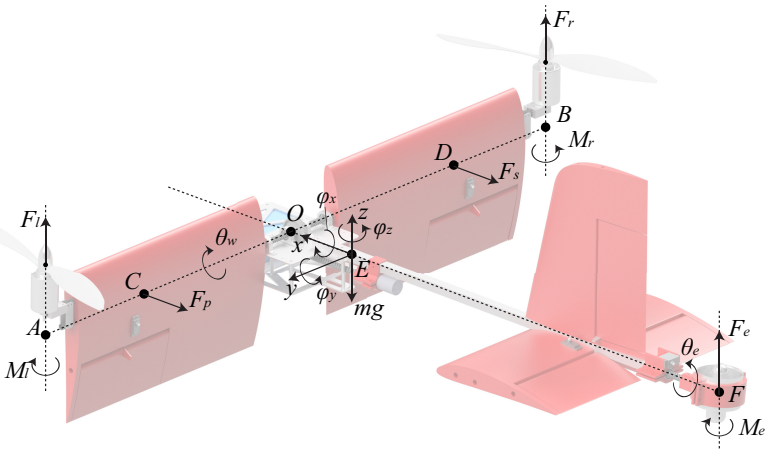

Fig. 4. Acting force diagram of the TWUAV in hovering state. $F_{l}, F_{r}$, and $F_{e}$ are the forces produced by the port and starboard motors, and the EDF, $F_{p}$ and $F_{s}$ are the forces produced by air flow over the wing profile, $M_{l}$, $M_{r}$, and $M_{e}$ are momentum caused by the spinning propellers or fan, $m g$ is the force caused by gravitational acceleration and mass, $\theta_{w}$ is the angle of the wing to the body (zero along the $x$-axis), $\theta_{e}$ is the angle of the EDF from the $z$-axis, and the capital letter $(O$ and $A$ through $F$ ) denote points of interest so that distances can be indicated. The COM is in $E$. 


$$
\left[\begin{array}{c}
F_{x} \\
F_{y} \\
F_{z} \\
\tau_{x} \\
\tau_{y} \\
\tau_{z}
\end{array}\right]=\left[\begin{array}{c}
-\left(F_{s}+F_{p}\right) \cdot s \theta_{w}+\left(F_{l}+F_{R}\right) \cdot c \theta_{w} \\
F_{e} \cdot s \theta_{e} \\
\left(F_{r}+F_{l}\right) \cdot s \theta_{w}+F_{e} \cdot c \theta_{e}+\left(F_{s}+F_{p}\right) \cdot c \theta_{w} \\
\left(F_{l}-F_{r}\right) \cdot s \theta_{w} \cdot(A O)+\left(M_{r}-M_{l}\right) \cdot c \theta_{w}+ \\
+\left(F_{p}-F_{s}\right) \cdot c \theta_{w} \\
-\left(F_{l}+F_{r}\right) \cdot s \theta_{w} \cdot(O E)+F_{e} \cdot c \theta_{e} \cdot(E F)- \\
-M_{e} \cdot s \theta_{e} \\
F_{e} \cdot s \theta_{e} \cdot(E F)+\left(M_{r}-M_{l}\right) \cdot s \theta_{w}-M_{e} \cdot c \theta_{e}+ \\
+\left(F_{p}-F_{s}\right) \cdot s \theta_{w}+\left(F_{e}-F_{l}\right) \cdot c \theta_{w} \cdot(A O)
\end{array}\right]+\mathbf{R} m g
$$

where $\sin \theta=s \theta$ and $\cos \theta=c \theta,(\mathrm{AB})$ is the (distance between point $\mathrm{A}$ and point $\mathrm{B}$ ), and $\mathbf{R}_{\mathbf{6 x} 6}$ defined as:

$$
\mathbf{R}=\left[\begin{array}{ll}
\mathbf{R}_{\text {rot }} & \mathbf{0}_{3 \times 3} \\
\mathbf{0}_{3 \times 3} & \mathbf{0}_{3 \times 3}
\end{array}\right]
$$

where $\mathbf{R}_{\text {rot }}$ is a three by three rotational matrix, representing the angular position of the body in relation to the direction of the gravitational acceleration.

At this point it should be highlighted that the additional aerodynamic forces exerted on the body and caused by movement through the air are neglected in the hovering state, where the angle of the wing $\theta_{w}$ will be fixed at 90 degrees. Furthermore, the forces produced by the flow of air from the propellers over the wing profile $-F_{s}$ and $F_{p}$ - are neglected. Thus, for the case of hovering, the above Equation (1) is simplified to:

$$
\left[\begin{array}{c}
F_{x} \\
F_{y} \\
F_{z} \\
\tau_{x} \\
\tau_{y} \\
\tau_{z}
\end{array}\right]=\left[\begin{array}{c}
0 \\
F_{e} \cdot s \theta_{e} \\
F_{r}+F_{l}+F_{e} \cdot c \theta_{e} \\
\left(F_{l}-F_{r}\right) \cdot(A O) \\
-\left(F_{l}+F_{r}\right) \cdot(O E)+F_{e} \cdot c \theta_{e} \cdot(E F)-M_{e} \cdot s \theta_{e} \\
F_{e} \cdot s \theta_{e} \cdot(E F)+M_{r}-M_{l}-M_{e} \cdot c \theta_{e}
\end{array}\right]+\mathbf{R} m g .
$$

In continuation, the system model can be derived by using the Newton-Euler kinematics for a rigid body[9]. Thus the Newton-Euler equations are formulated as:

$$
\left[\begin{array}{c}
F \\
\tau
\end{array}\right]=\left[\begin{array}{cc}
m I_{3 \times 3} & 0 \\
0 & I_{c m}
\end{array}\right]\left[\begin{array}{c}
a_{c m} \\
\dot{\omega}
\end{array}\right]+\left[\begin{array}{c}
0 \\
\omega \times\left(I_{c m} \omega\right)
\end{array}\right],
$$

where $F=\left[\begin{array}{lll}F_{x} & F_{y} & F_{z}\end{array}\right]^{T} \in \mathbb{R}^{3}$ is the force acting on the COM, $\tau=\left[\begin{array}{lll}\tau_{x} & \tau_{y} & \tau_{z}\end{array}\right]^{T} \in \mathbb{R}^{3}$ the torque around the COM, $\omega=\left[\begin{array}{lll}\omega_{x} & \omega_{y} & \omega_{z}\end{array}\right]^{T} \in \mathbb{R}^{3}$ the angular velocity, $a_{c m}=$ $\left[\begin{array}{lll}a_{x} & a_{y} & a_{z}\end{array}\right]^{T} \in \mathbb{R}^{3}$ the acceleration of COM, $I_{3 \times 3}$ is the $3 \times 3$ identity matrix and the inertia matrix $I_{c m}$, defined as:

$$
I_{c m}=\left[\begin{array}{ccc}
I_{x x} & I_{x y} & I_{x z} \\
I_{y x} & I_{y y} & I_{y z} \\
I_{z x} & I_{z y} & I_{z z}
\end{array}\right] \in \mathbb{R}^{3 x 3}
$$

The Newton-Euler equations in Equation (4), solved for the acceleration and the angular acceleration yields:

$$
\left[\begin{array}{c}
a_{c m} \\
\dot{\omega}
\end{array}\right]=\left[\begin{array}{cc}
m I_{3 \times 3} & 0 \\
0 & I_{c m}
\end{array}\right]^{-1}\left[\begin{array}{c}
F \\
\tau
\end{array}\right]-\left[\begin{array}{cc}
m I_{3 \times 3} & 0 \\
0 & I_{c m}
\end{array}\right]^{-1}\left[\begin{array}{c}
0 \\
\omega \times\left(I_{c m} \omega\right)
\end{array}\right]
$$

As has been shown in [10], both the force and the momentum produced by the propellers are proportional to the square of the input signal to the motor. Consequently, the forces exerted by the motors as well as the momentum from the rotating propellers are modelled as a constant times the square of the input signal as follows:

$$
\begin{aligned}
F_{i} & =A_{F, i} \cdot \widetilde{u}_{i}^{2} \\
M_{i} & =A_{M, i} \cdot \widetilde{u}_{i}^{2} \\
\widetilde{u}_{i} & =\frac{1}{\tau_{s, i}+1} \cdot u_{i}
\end{aligned}
$$

where $F_{i}$ and $M_{i}$ are the force and momentum exerted by the propeller respectively, $A_{F, i}$ and $A_{M, i}$ are the force and momentum constants, $\tau_{s, i}$ the time constant for the motors, and $u_{i}\left(0 \leqslant u_{i} \leqslant 1\right)$ for $i=1,2,3$, defines the signals to the motors and servo. Since the left and right propeller motors will have the same physical properties, they will have the same force constant $A_{F, r}$ and momentum constant $A_{M, r}$, but different input signals. The signal notations will be $u_{1}$, $u_{2}$, and $u_{3}$ for the port motor, starboard motor and EDF respectively. The angle of the EDF servo can be defined as:

$$
\theta_{e}=\frac{\pi}{3} \cdot u_{4}, \quad-1 \leqslant u_{4} \leqslant 1
$$

where $u_{4}$ is the signal to the servo.

The final hovering model calculated from (3) and (5) can then be derived as:

$$
\left[\begin{array}{c}
a_{x} \\
a_{y} \\
a_{z} \\
\dot{\omega}_{x} \\
\dot{\omega}_{y} \\
\dot{\omega}_{z}
\end{array}\right]=\left[\begin{array}{c}
0 \\
s \theta_{e} \cdot A_{F, e} \frac{u_{3}^{2}}{m} \\
\frac{A_{F, r} \cdot\left(\widetilde{u}_{2}^{2}+\widetilde{u}_{1}^{2}\right)+c \theta_{e} \cdot A_{F, e} \cdot \frac{\widetilde{u}_{3}^{2}}{m}}{\frac{A_{F, r} \cdot\left(\widetilde{u}_{1}^{2}-\widetilde{u}_{2}^{2}\right) \cdot(A O)}{I_{x x}+I_{x y}+I_{x z}}-\omega_{x}^{2}} \\
\frac{A_{F, e} \cdot \widetilde{u}_{3}^{2} \cdot s \theta_{e} \cdot(E F)+(O E)+A_{F, e} \cdot \widetilde{u}_{e}^{2} \cdot c \theta_{e} \cdot(E F)-A_{M, e} \cdot \widetilde{u}_{e}^{2} \cdot s \theta_{e}}{I_{M, r}+I_{y}+I_{y z}-A_{M, r} \cdot \widetilde{u}_{1}^{2}-A_{M, e} \cdot \widetilde{u}_{3}^{2} \cdot c \theta_{e}}-\omega_{y}^{2} \\
I_{z x}+I_{z y}+I_{z z}
\end{array}\right]+\mathbf{R} g .
$$

where $\widetilde{u}_{1,2,3}$ are defined according to (8) and the angle $\theta_{E}$ defined in (9).

\section{Control Scheme}

For the preliminary evaluation of the TW-UAV's hovering capabilities, a control scheme based on the utilization of $\mathrm{P}$, PI and PID controllers was used as indicated in Figure 5. In particular, for the attitude control, a cascade P-PI controller (Fig. 6) was implemented, where three separate PI controllers were used to regulate the angular velocities around the $x$ (roll), $y$ (pitch), and $z$ (yaw) axes, while the reference angle is fed through a $\mathrm{P}$ regulator for extracting the reference for the angular velocity. Figure 5 presents the whole control system architecture and Figure 6 shows the specifics of the P-PI controller.

The output values of the controllers are directed though a Signal Mixer (SM) before being used as inputs to the motors and the servo. The output of the roll controller is subtracted from one propeller motor and added to the other one, depending on which way the UAV needs to roll.

Similarly, the pitch control output is either subtracted from the EDF signal and added to both the propeller motors or vice versa. For the yaw control the output is directly sent as an input signal to the servo controlling the angle of the EDF. In equation form the corresponding signals can be defined 


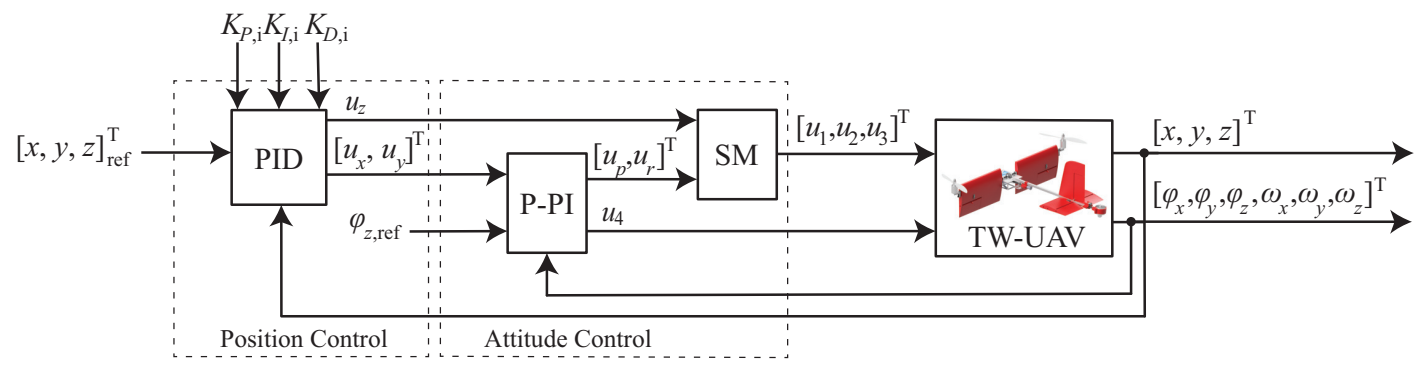

Fig. 5. Position and attitude control structure for the TWUAV.

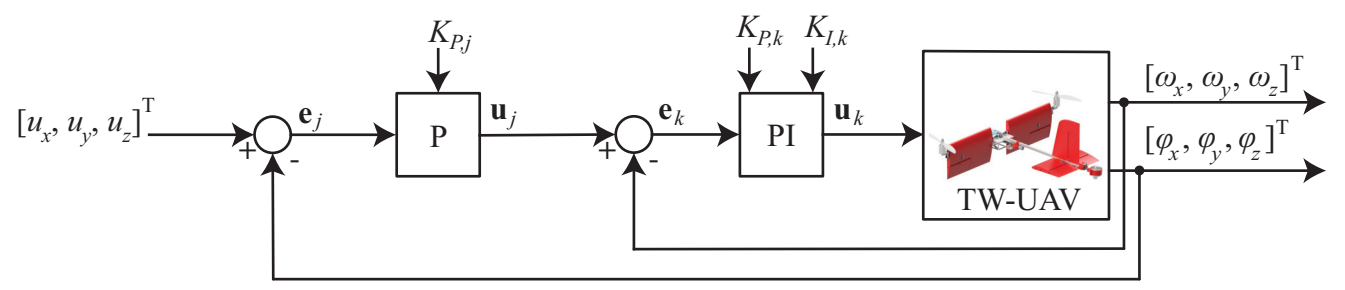

Fig. 6. P-PI structure for the attitude control for the TWUAV.

as follows:

$$
\begin{aligned}
& u_{1}=u_{z}+u_{r}-u_{p} \\
& u_{2}=u_{z}-u_{r}-u_{p} \\
& u_{3}=u_{z}+u_{p}
\end{aligned}
$$

Three PID loops were implemented to control position, since only the position is assumed to be observable. This assumption is based on the idea that hovering will be tested in a camera lab or other position tracking system. Outputs of $x$-position and $y$-position control are directly inserted as references to the roll and pitch P-PI controllers, and the height control output is inserted as a throttle signal to both propeller motors and to the EDF.

\section{Simulation Results}

To evaluate the TW-UAV, a series of simulations were made. These included a set of position step reference responses, a path following simulation, and a torque disturbance simulation. The angular references in all the simulations were set to zero, i.e. the attitude coordinates of the aircraft would always be the same as those of the frame's. Sensor noise disturbance was added to all the outputs in the simulation to observe the controllers' ability to handle noise. Table I shows the size of the noise added to the outputs. It should be noted that all the simulations were run with a sample rate of $50 \mathrm{~Hz}$.

TABLE I

NOISE Disturbance

\begin{tabular}{|c|c|}
\hline Output & Amplitude of random noise \\
\hline \hline Position & $0.001^{2} \mathrm{~m}$ \\
\hline Angular Velocity & $0.17^{2} \mathrm{rad} / \mathrm{s}$ \\
\hline Body Angle & $0.0087^{2} \mathrm{rad}$ \\
\hline
\end{tabular}

In these simulations, a Computer Aided Design (CAD) programme was used to produce the inertia matrix $I_{c m}$. In this approach, proper design materials were added to the different parts in the CAD model so that the weight, centre of mass, and inertia matrix could be estimated for use in the simulations. In the calculations, the wings and holders for motors and servos were considered to be designed with a PLA plastic with 20 percent infill, which is a reasonable density for this type of structure[3]. The wings' profile was the same as in [3],i.e. NACA4412, but scaled from a $0.15 \mathrm{~m}$ chord (distance from the front of the wing to the back) to a $0.25 \mathrm{~m}$ chord. This allows the wingspan to be shortened from a $1.58 \mathrm{~m}$ wingspan to $1.00 \mathrm{~m}$ for more mechanical stability. In addition, the tubes strengthening the wings and the drive shaft for the worm drive were set to carbon fiber in the design. The chassis and other flat structural parts were set as aluminium, and lastly the motors, battery, and servos were given masses corresponding to commercially available products of their intended size.

In Table II the parameters and the applicable dimensions of the simulated aircraft are shown. Most parameters are taken from the CAD model except for the weight and motor constants. The weight has a reasonable margin added to the weight calculated by the CAD software for cables and electronics and the motor constants are estimated from commercially available motors and EDFs. The control parameters utilized in the proposed control scheme in Section IV are displayed in Table III.

In Figure 7 the aircraft has a constant position reference but disturbance torques are added around each of the main axes to observe the attitude control and response. In the onehundred seconds simulation, a $3 \mathrm{Nm}$ torque pulse is added around the $x$-axis at $30 \operatorname{seconds}(d 1)$, a $3 \mathrm{Nm}$ torque pulse is added around the $y$-axis at $45 \operatorname{seconds}(d 2)$, and a $5 \mathrm{Nm}$ torque pulse disturbance around the $z$-axis at 60 seconds $(d 3)$. The corresponding control signals to the motors and servo 
TABLE II

DESIGN PARAMETERS OF THE TWUAV

4

\begin{tabular}{|c|c|c|}
\hline Parameter & Value & Unit \\
\hline \hline$(\mathrm{OE})$ & 0.15 & $\mathrm{~m}$ \\
\hline$(\mathrm{OA}),(\mathrm{OB})$ & 0.56 & $\mathrm{~m}$ \\
\hline$(\mathrm{EF})$ & 0.745 & $\mathrm{~m}$ \\
\hline $\mathrm{m}$ & 4.9 & $\mathrm{Kg}$ \\
\hline$I_{x x}$ & $433.6 \cdot 10^{-} 3$ & $\mathrm{Kg} \cdot \mathrm{m}^{2}$ \\
\hline$I_{y y}$ & $528.8 \cdot 10^{-} 3$ & $\mathrm{Kg} \cdot \mathrm{m}^{2}$ \\
\hline$I_{z z}$ & $921.6 \cdot 10^{-} 3$ & $\mathrm{Kg} \cdot \mathrm{m}^{2}$ \\
\hline$I_{x y}, I_{y x}$ & $7.467 \cdot 10^{-} 3$ & $\mathrm{Kg} \cdot \mathrm{m}^{2}$ \\
\hline$I_{x z}, I_{z x}$ & $22.573 \cdot 10^{-} 3$ & $\mathrm{Kg} \cdot \mathrm{m}^{2}$ \\
\hline$I_{y z}, I_{z y}$ & $-0.938 \cdot 10^{-} 3$ & $\mathrm{Kg} \cdot \mathrm{m}^{2}$ \\
\hline$A_{F, L}, A_{F, R}$ & 30 & $\mathrm{~N}$ \\
\hline$A_{F, E}$ & 20 & $\mathrm{~N}$ \\
\hline$A_{M, L}, A_{M, R}, A_{M, E}$ & 1 & $\mathrm{~N} \cdot \mathrm{m}$ \\
\hline$\tau_{s, 1}, \tau_{s, 2}$ & 0.15 & $\mathrm{~s}$ \\
\hline$\tau_{s, 3}$ & 0.2 & $\mathrm{~s}$ \\
\hline \multicolumn{2}{|c}{}
\end{tabular}

TABLE III

CONTROL PARAMETERS OF THE TWUAV

\begin{tabular}{|c|c|c|c|}
\hline Gain & \multicolumn{3}{|c|}{ Values $(x, y, z)$} \\
\hline \hline$K_{P, i}$ & 0.09 & 0.05 & 1 \\
\hline$K_{I, i}$ & 0.05 & 0.03 & 0.35 \\
\hline$K_{D, i}$ & 0.2 & 0.03 & 1 \\
\hline$K_{P, j}$ & 1 & 1.4 & 1 \\
\hline$K_{P, k}$ & 0.05 & 0.07 & 1 \\
\hline$K_{I, k}$ & 0.08 & 0.06 & 1 \\
\hline
\end{tabular}

can be seen in Figure 8.

From the obtained results it is obvious that the proposed scheme can perform a very good tracking of a varying reference, even in the case of intense and multiple external disturbances that can cause significant deterioration of the overall performance of the closed loop system, and even drive it to instabilities (crashes). Additionally it should be mentioned that the proposed controller can achieve a rapid convergence to the reference signal, without intense overshoots and oscillations. After the occurrence of a disturbance, the TW-UAV manages to perform a quick recovery in tracking, of only a few seconds in duration, a characteristic that is much desirable in flying under windy conditions.

In Figure 9 the TW-UAV's response to a changing position reference is simulated, while in Figure 10 the corresponding three dimensional representation of the reference signals and response are depicted for a better understanding of the reference input in relation to the achieved tracking response. As can been seen from the results, both the $x$ and $y$ position responses can be interpreted as slow due to the cascaded nature of the controller, while the proposed scheme is able again to track very satisfactorily the desired position commands with fast settling times and without intense oscillations. As a final comparison factor, the Root Mean Square Error (RMSE) of the reference and achieved response for the commanded translation from Figure 9 is depicted in Table IV.

As a general remark for the presented simulation results,
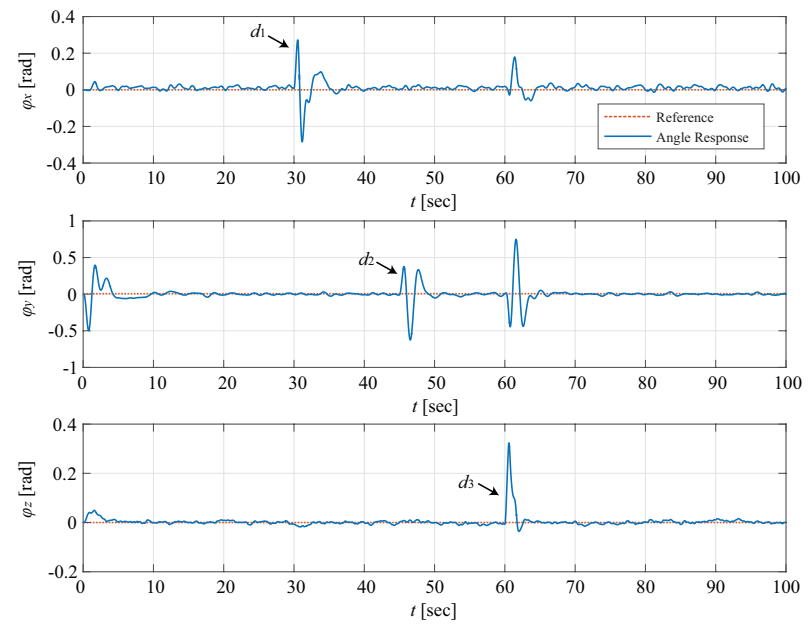

Fig. 7. Simulated response of the TW-UAV to a constant position reference. Disturbance torques are applied in random intervals to test its hovering capabilities.
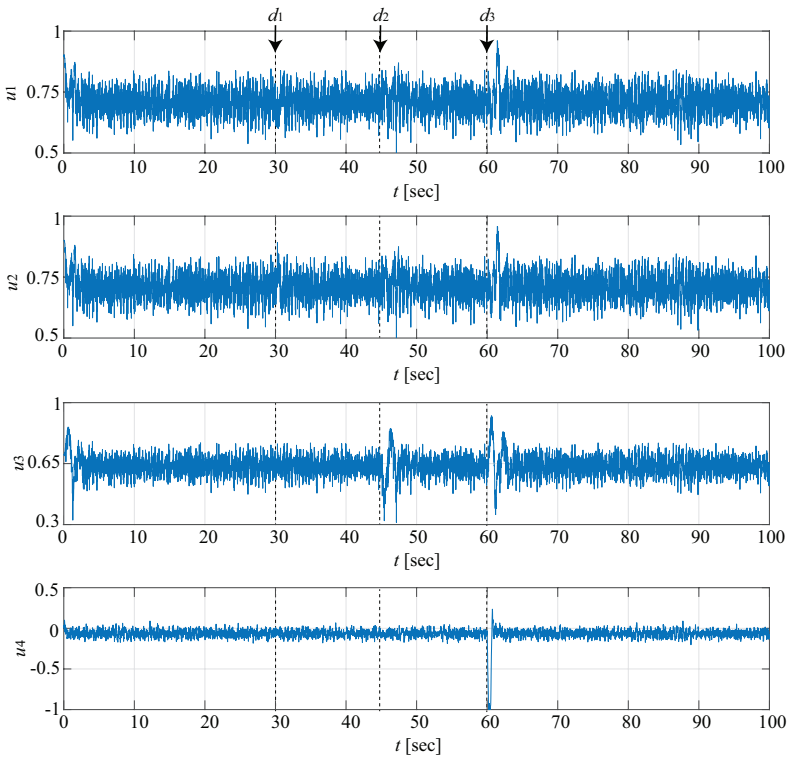

Fig. 8. Corresponding control effort signals to the TW-UAV motors for the simulation presented in Figure 7.
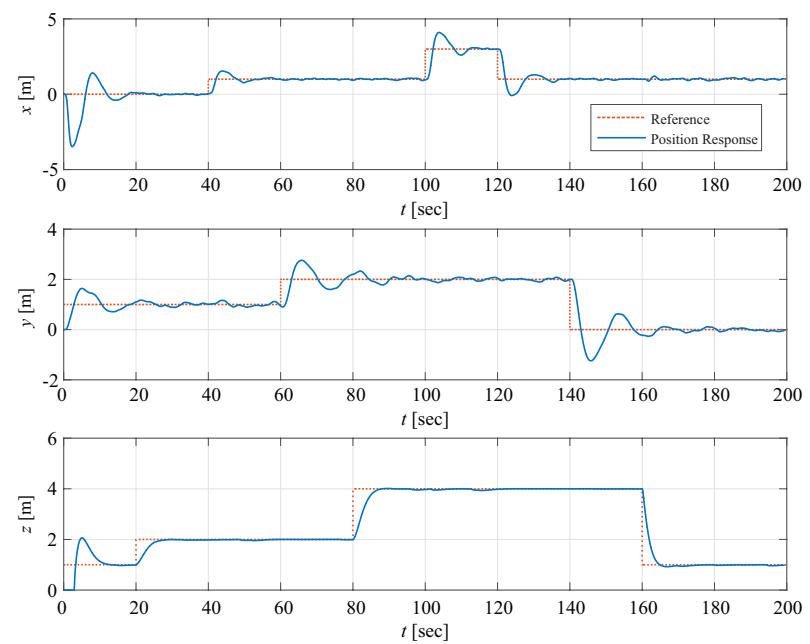

Fig. 9. Step reference and response for position in $x, y$, and $z$. 


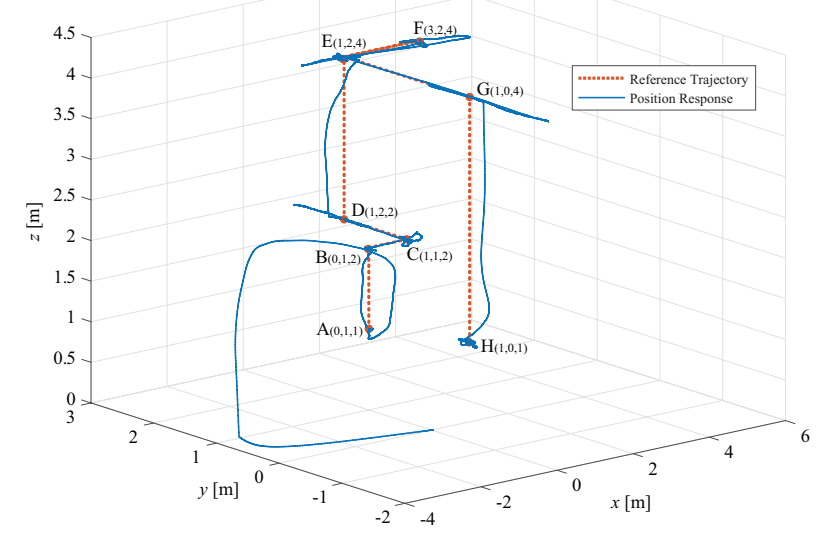

Fig. 10. 3D representation of the reference signals and responses from Fig. 9

TABLE IV

Root MeAn SQUare ERror IN Fig. 9

\begin{tabular}{c|c} 
RMSE & Value \\
\hline \hline Error in $x$ & $0.3382 \mathrm{~m}$ \\
\hline Error in $y$ & $0.3524 \mathrm{~m}$ \\
\hline Error in $z$ & $0.3071 \mathrm{~m}$
\end{tabular}

it should be stated that for the first 20 seconds of simulation the lack of simulated floor, the lack of consideration of the ground effect, and the different start-up times for the motors, cause the TW-UAV to diverge slightly from both the reference and the starting position. For this reason, the following simulation will highlight the TW-UAV's response behavior once it has started up, taken off and settled at 0.5 meters above the ground. Figure 11 shows a position reference and the corresponding position response, as in the previous simulation, but with the first twenty five seconds erased, in order to avoid considering the effects of the transient response. As in the previous scenarios considered, the response of the UAV is very fast and accurate, while due to sudden variations of the reference, an overshoot-like response at the corresponding time instances is observed but with a rapid settling time and with no oscillations. Finally, in Table V the RMSE between the reference and the obtained response for this simulation is being presented.

TABLE V

Root Mean Square ERror IN Fig. 11

\begin{tabular}{c|c} 
RMSE & Value \\
\hline \hline Error in $x$ & $0.1194 \mathrm{~m}$ \\
\hline Error in $y$ & $0.2151 \mathrm{~m}$ \\
\hline Error in $z$ & $0.0322 \mathrm{~m}$
\end{tabular}

\section{CONCLUSIONS}

In this article the design, modelling and control of a novel Tilt-Wing UAV has been considered in simulation studies.

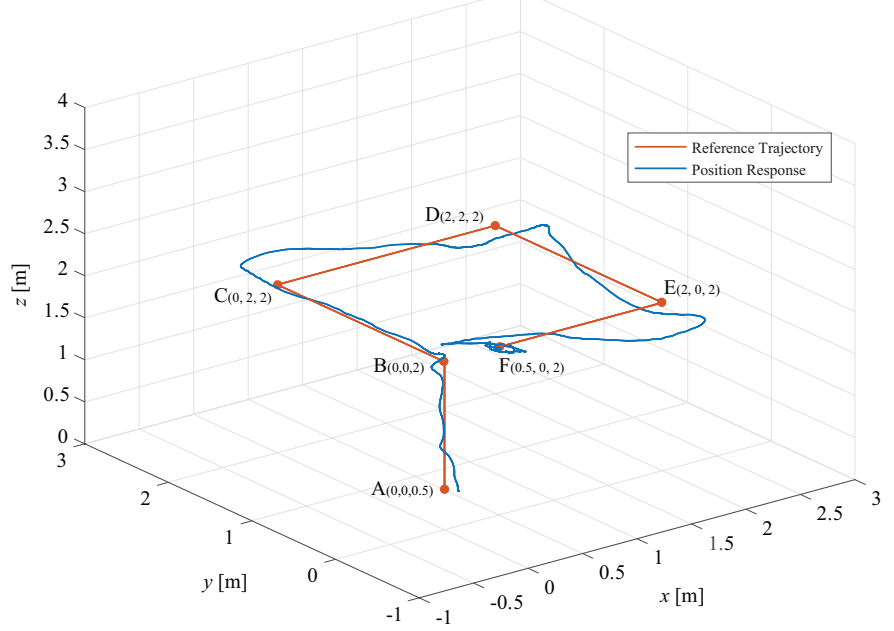

Fig. 11. Position reference response of the TWUAV. The aircraft is in position $\mathrm{A}$ at 25 seconds after the simulation start.

The main conclusion to be drawn from these simulations is that the design of the physical model is viable for further experimental development. Considering the TW-UAV's size and simple actuators, the aircraft was able to follow the references well and sustain its attitude easily even though only a conventional control scheme was used. The attitude responses showed that having a P-PI controller for attitude control is indeed suitable, since the responses were fast and robust even though the added torques were large. For the position control, the cascaded PIDs were rather slow in responding to the fast changes in reference, a fact that indicates the need for a faster control scheme development or a further tuning of the existing one.

\section{REFERENCES}

[1] E. Fresk and G. Nikolakopoulos, "Full quaternion based attitude control for a quadrotor," in European Control Conference, July 2013.

[2] K. Alexis, G. Nikolakopoulos, A. Tzes, and L. Dritsas, "Coordination of helicopter uavs for aerial forest-fire surveillance," in Applications of intelligent control to engineering systems. Springer, 2009, pp. 169-193.

[3] A. Lindqvist, E. Fresk, and G. Nikolakopoulos, "Optimal design and modeling of a tilt wing aircraft," in Control and Automation (MED), 2015 23th Mediterranean Conference on. IEEE, 2015, pp. 701-708.

[4] C. Papachristos, K. Alexis, G. Nikolakopoulos, and A. Tzes, "Model predictive attitude control of an unmanned tilt-rotor aircraft," in IEEE International Symposium on Industrial Electronics, 2011, pp. 922-927.

[5] A. Kahvecioglu and N. Alemdaroglu, "Optimal design of a miniature quad tilt rotor uav," in Unmanned Aircraft Systems (ICUAS), 2015 International Conference on, June 2015, pp. 1118-1127.

[6] F. Senkul and E. Altug, "Adaptive control of a tilt - roll rotor quadrotor uav," in Unmanned Aircraft Systems (ICUAS), 2014 International Conference on, May 2014, pp. 1132-1137.

[7] K. Cesare, R. Skeele, S.-H. Yoo, Y. Zhang, and G. Hollinger, "Multiuav exploration with limited communication and battery," in Robotics and Automation (ICRA), 2015 IEEE International Conference on, May 2015, pp. 2230-2235.

[8] F. Irving, An Introduction to the Longitudinal Static Stability of LowSpeed Aircraft. Oxford, UK: Pergamon Press, 1966.

[9] H. Hahn, Rigid Body Dynamics of Mechanisms. Berlin Heidelberg: Springer-Verlag, 2002.

[10] E. Fresk and G. Nikolakopoulos, Experimental Model Derivation and Control of a Variable Pitch Propeller Quadrotor, ser. IEEE International Conference on Control Applications. Proceedings. IEEE, 2014, pp. 723-729. 